\begin{tabular}{|c|l|}
\hline Title & Power-Law Stress and Creep Relaxations of Single Cells Measured by Colloidal Probe A tomic Force Microscopy \\
\hline Author(s) & $\begin{array}{l}\text { Hiratsuka, Shinichiro; Mizutani, Y Ysuke; Toda, A kitoshi; Fukushima, Norichika; Kaw ahara, Koichi; Tokumoto, } \\
\text { Hiroshi; Okajima, Takaharu }\end{array}$ \\
\hline Citation & $\begin{array}{l}\text { Japanese Journal of A pplied Physics, 48(8), 08JB17 } \\
\text { https://doi.org/40.1143/JAAP.48.08JB17 }\end{array}$ \\
\hline Issue Date & 2009-08 \\
\hline Doc URL & http://hdl.handle.net/2115/45747 \\
\hline Type & article (author version) \\
\hline File Information & JJA P48_08JB17.pdf \\
\hline
\end{tabular}

Instructions for use 


\section{Power-Law Stress and Creep Relaxations of Single Cells Measured by Colloidal Probe Atomic Force Microscopy}

Shinichiro HIRATSUKA, Yusuke MIZUTANI, Akitoshi TODA ${ }^{1}$, Norichika FUKUSHIMA $^{1}$, Koichi KAWAHARA, Hiroshi TOKUMOTO ${ }^{2}$, and Takaharu OKAJIMA*

Graduate School of Information Science and Technology, Hokkaido University, Sapporo 060-0814, Japan

${ }^{1}$ MEMS Technology Division, Olympus Corporation, Tokyo 192-8512, Japan

${ }^{2}$ Nanotechnology Research Center, Research Institute for Electronic Science, Hokkaido University, Sapporo 001-0021, Japan

*E-mail address: okajima@ist.hokudai.ac.jp 


\begin{abstract}
We measured stress and creep relaxations of mouse fibroblast cells arranged and cultured on a microarray, by colloidal probe atomic force microscopy (AFM). A hydrophobic monolayer coating of perfluorodecyltrichlorosilane (FDTS) on the surface of colloidal silica beads significantly reduced the adhesion force of live cells, compared with untreated beads. The rheological behaviors of cells were estimated by averaging several relaxation curves of cells measured by the AFM. Longer-time tailing of both stress and creep relaxation curves followed single power-law behavior over a time scale of $60 \mathrm{~s}$, with exponents in the range $0.1-0.4$, varying with cells. The results were in good agreement with previous measurements of the frequency-domain rheology of cells using the force modulation mode.
\end{abstract}




\section{Introduction}

The rheology of living cells plays a key role in determining cell functions such as motility, division and adhesion. The micro- and nano-rheological properties of living cells have been extensively investigated with various methods such as magnetic twisting cytometry (MTC) ${ }^{1-6)}$, laser tracking micro-rheology (LTM) ${ }^{4-7)}$, and atomic force microscopy (AFM) ${ }^{8-20)}$. AFM offers the significant advantages of being able to control the position of the AFM probe and not requiring modification of the cell surface.

Recently, the complex shear modulus of cells in the frequency domain has been measured using the force modulation mode of AFM ${ }^{10-15)}$, which measures the dynamic response of the loading force to an external periodic strain. Moreover, by combining the force modulation mode with microarray techniques, we measured ${ }^{16)}$ the distribution of the complex shear modulus of a large number of cells $(n=130)$ arranged and cultured on a microarray. These AFM studies revealed that the averaged complex shear modulus of cells followed a power-law regime, such as the structural damping model ${ }^{21,22)}$, which is considered to be governed by so-called soft glass rheology (SGR) ${ }^{1,2)}$.

On the other hand, using time domain AFM experiments, $\mathrm{Wu}$ et al. ${ }^{17)}$ demonstrated, for the first time, a creep test for cells. In this measurement, the loading force was held constant by controlling the cantilever base displacement and the displacement was then measured as a function of time. Darling et al. ${ }^{18,19)}$ measured the stress relaxation of cells by holding the cantilever base displacement constant and measuring the loading force as a function of time. In these time-domain AFM studies, the relaxations observed over a timescale of $60 \mathrm{~s}$ were estimated by the standard linear 
solid (SLS) model, in which cells are assumed to consist of two elastic elements and a viscous element. Moreover, it was argued that the stress relaxations of cells ${ }^{20,21)}$ over short timescales of less than a few seconds were well-fitted by a stretched exponential function, rather than the single exponential function expected for the SLS model. However, it has not yet been determined how stress or creep relaxations compare with the power-law regime of the complex shear modulus observed by the force modulation mode ${ }^{10-15)}$. Thus the characteristics of the rheological behavior of cells, as determined by AFM measurements, are still under debate.

Determination of the precise functional form of the time-domain relaxations of cells may require measurement of the averaged relaxation curves of cells, similar to the case in the frequency-domain, where the functional form of the rheology can be estimated by averaging the measurement results ${ }^{2,3,6,7,16)}$. Therefore, in the present study, we measured several stress and creep relaxations of individual cells over a time interval of $60 \mathrm{~s}$ and from these estimated the averaged relaxations of each cell. It was found that the averaged relaxation curves clearly exhibited power-law behavior. The results were compared with those obtained in previous studies using MTC and AFM in the frequency domain.

\section{Experimental Procedure}

\subsection{Cell samples}

The cell preparation has been described in detail elsewhere ${ }^{16,24)}$. Briefly, mouse fibroblast (NIH3T3) cells were arranged with a filling rate of $>98 \%$ in the wells of a 
commercial cell microarray (Nunc Livecell Array), the substrate of which had been precoated with fibronectin (BD Bioscience). Cells were then cultured in Dulbecco's modified Eagle's medium (Sigma DMEM) containing fetal bovine serum (FBS) at $37{ }^{\circ} \mathrm{C}$ in a $5 \% \mathrm{CO}_{2}$ atmosphere. Prior to AFM measurements, the cell samples were washed with $\mathrm{CO}_{2}$-independent medium (GIBCO-BRL Life Technologies). AFM measurements were conducted in the same medium at room temperature [Fig. 1(a)].

\subsection{AFM measurements}

A commercial AFM (Asylum Research MFP-3D AFM) was mounted in an inverted optical microscope (Nikon TE-2000E). A small rectangular cantilever (Olympus BL-AC40TS) with a nominal spring constant of less than $0.1 \mathrm{~N} / \mathrm{m}$ was used. After a colloidal silica bead with a radius of ca. $5 \mu \mathrm{m}$ (Funakoshi) was attached to the apex of the AFM tip ${ }^{16,21,24)}$ using an epoxy glue, a perfluorodecyltrichlorosilane (FDTS) film ${ }^{25,26)}$ was deposited as a self-assembled monolayer (SAM) onto the surface of colloidal silica by a vapor process. The water contact angle on flat glass substrates on which FDTS had been deposited by the same process was ca. $110^{\circ}$. The spring constant of the cantilever was determined using a thermal fluctuation method ${ }^{27)}$. The loading force was calculated, using Hooke's law, by multiplying the calibrated cantilever spring constant by its deflection.

During stress relaxation measurements, the cantilever base displacement was kept constant at the position where the initial trigger force reached approximately 500 $\mathrm{pN}$, which corresponded to be $1-2 \mu \mathrm{m}$ of indentation, and the loading force was 
measured as a function of time. Since, in the stress relaxation process, the change in deflection of cantilever was ca. $10 \mathrm{~nm}$, which was $\sim 1 \%$ with respect to the indentation, the indentation was regarded as approximately constant in time. On the other hand, during creep measurements, the loading force was kept at a constant value of $300 \mathrm{pN}$ using a feedback circuit, and the position of the cantilever displacement was monitored as a function of time. As shown in Fig. 1(b), we first measured several stress relaxation curves for each of five cells at the center of the microarray wells. The creep measurements were then obtained for the same cells. The stress and creep relaxation curves obtained from the single cells were normalized, and the averaged relaxation curves were estimated for the cells.

\subsection{Stress and Creep relaxations measured by colloidal probe AFM}

When a spherical colloidal probe with a radius $R$ indents a cell surface with viscoelastic properties, the loading force, $F$, at time, $t$, with indentation, $\delta$, is approximately given using a Hertz model, ${ }^{28)}$ by

$$
F(t)=\frac{4 R^{1 / 2} E(t)}{3\left(1-v^{2}\right)} \delta^{3 / 2}(t)
$$

where $E$ is the relaxation modulus at $t$, and $v$ is the Poisson's ratio of the cell, which was assumed to be $0.5^{8)}$. Here, we assume that the shear modulus of cells, $G(f)$, follows a single power-law function of frequency, $f$, of the form $f^{\alpha \quad 1-6,21,22)}$. 
In the case of stress relaxation with a constant indentation $\delta_{0}, F(t)$ is proportional to $E(t) H(t)$, using the Heaviside step function $H(t){ }^{18,29)}$. Since the relaxation modulus in the Laplace domain, $E(f)$, is proportional to $f^{\alpha}$, by using the relation $E(f)=2(1+v) G(f)^{28)}$, the loading force in the Laplace domain, $F(f)$, is proportional to $f^{\alpha-1}$. Therefore, the inverse Laplace transform of $F(f)$ yields the functional form of loading force for stress relaxation

$$
F(t) \propto t^{-\alpha}
$$

Therefore, the averaged stress relaxation curve of the cell was fitted by

$$
F(t)=p t^{-\alpha}+q
$$

where $p$ and $q$ are fitting parameters.

In the case of creep relaxation with a constant loading force, the relation between the contact radius $a(t)$ and the creep compliance $J(t)$ becomes $a^{3}(t) \propto J(t){ }^{17,30)}$. Since $E(t) \propto t^{-\alpha}, J(t)$ is proportional to $t^{\alpha}$. Moreover, using the relation $a^{2}(t)=R \delta{ }^{28)}$, we finally obtain the functional form of indentation for creep relaxation

$$
\delta(t) \propto t^{2 \alpha / 3}
$$

Therefore, the averaged creep relaxation curve of the cell was fitted by using

$$
\delta(t)=p t^{2 \alpha / 3}+q
$$

\section{Results and Discussion}

Figure 2 shows the distribution of adhesion work between the colloidal probe and NIH3T3 cells in force curve measurements at a trigger force of $1.0 \mathrm{nN}$. Clearly, the adhesion work measured using the FDTS-coated silica probe was significantly lower than that measured with untreated silica probes: the average adhesion work with and 
without FDTS-treated probe being $0.057 \mathrm{fJ}$ and $0.12 \mathrm{fJ}$, respectively. In the Hertz model of eq. (1), which has been widely employed to estimate the mechanics of cells ${ }^{8)}$, it is assumed that there is no adhesion between contacting materials. Moreover, reduced adhesion prevents damage to cells and their detachment from the substrate. Therefore, we concluded that the FDTS-coated silica probe was more suitable than untreated probes for measuring cellular mechanics. As shown in the insets of Fig. 2, no correlation was observed between the adhesion work and time for either type of probe the adhesion work was randomly distributed over time, i.e., randomly distributed between cells.

Figure 3(a) shows a typical curve of the averaged stress relaxation of NIH3T3 cells cultured in the wells of the microarray, as measured by AFM. It can be seen that the loading force decreased sharply within several seconds. This relaxation could not be fitted well by a single exponential function, in agreement with previous reports ${ }^{20,21)}$. Furthermore, the loading force continued to decrease gradually over the remainder of the measurement time, without attaining an equilibrium state. As shown in the inset of Fig. 3(a), the functional form of the stress relaxation followed power-law behavior. Therefore, we estimated the power-law exponent of the averaged stress relaxation curves using eq. (2').

Figure 3(b) shows a typical curve of the averaged creep relaxation of NIH3T3 cells cultured in the wells of the microarray, measured by AFM. Similar to the stress relaxation behavior, the displacement of cantilever position initially increased sharply, over a period of several seconds, and then continued to increase gradually over the 
subsequent measurement time. As shown in the inset of Fig. 3(b), the longer-time portion of the creep curve $(>2 \mathrm{~s})$ followed power-law behavior but the curve did not follow the power-law regime over the first $2 \mathrm{~s}$. The latter behavior was probably due to the feedback error of creep tests, and thus we estimated the power-law exponent of the averaged creep relaxation curves over $2 \mathrm{~s}$ using eq. (3').

The power-law exponent, $\alpha$, of stress and creep relaxations estimated from the average curves for a number of individual cells $(n=25)$ is plotted in Fig. 4 . It can be seen that $\alpha$ is distributed in the range of $0.1-0.4$, depending on the cells. Interestingly, the $\alpha$ 's of stress and creep relaxations were not necessarily consistent for individual cells. Note that the indentation depth differed between stress and creep experiments. Specifically, in stress relaxation experiments, the indentation was fixed at $1-2 \mu \mathrm{m}$ during the measurements, whereas in creep experiments the indentation increased from $<1 \mu \mathrm{m}$ to a few $\mu \mathrm{m}$ over the time of the experiment. Therefore, differences between the $\alpha$ values obtained for stress and creep relaxations may be explained by heterogeneities in the cell structures that contribute to the observed relaxations. Another plausible explanation of the difference is that the cell mechanics fluctuate in time due to their viability.

In spite of the variations in the power-law exponent seen in both stress and creep relaxation measurements, the average values of $\alpha$ were almost the same for both relaxation types: $0.19 \pm 0.06$ for stress relaxations and $0.23 \pm 0.06$ for creep relaxations, respectively. The complex shear modulus, $G(f)$, of the NIH3T3 cells arranged and cultured on the same type of microarray used in the present study, has been measured as a function of frequency in the range $f=0.5-200 \mathrm{~Hz}$, using the force modulation mode ${ }^{16)}$. 
The results showed that $G$ for the cells in this frequency range exhibited power-law behavior with an exponent of $0.23 \pm 0.09$, which is in good agreement with that estimated from the average stress and creep relaxation curves shown in Fig. 4. This result suggests that the average rheological behavior of cells measured by AFM is identical in time regions from milliseconds to several tens seconds .

Some researchers have been proposed that the rheology involved in pulling single cells exhibits two power-law regimes in a long time period, rather than a single one. Desprat et al. ${ }^{31)}$ reported that the creep function of pulling a whole cell followed a power-law exponent of 0.24 for times less than $200 \mathrm{~s}$ while above $200 \mathrm{~s}$ the exponent increased to $\sim 0.5$. Overby et al. ${ }^{32)}$ reported that the power-law exponent of the creep of pulling a single cell was 0.18 (presumed to be governed by SGR) in the time range of several seconds and showed ca. 0.5 at timescales of more than several seconds. Moreover, Stamenovic et al. ${ }^{33)}$ showed a similar behavior of two power-law regimes with an intervening plateau in a time region of $\sim 10 \mathrm{~s}$. In our experiments, it appeared in some cases that both stress and creep relaxations exhibited time-dependent power-law behavior similar to that mentioned above. However, when we averaged several relaxation curves for each cell to obtain the average power-law exponent, the time-dependent power-law behavior almost vanished and only the single power-law behavior shown in Fig. 3 was exhibited, indicating that there was no existence of time-dependent power-law behavior in cellular rheology in the measurement time scale.

In the present study, single cells were placed in each of wells at their high concentration, so that they could not migrate freely on the substrate but fluctuate around 
the center of wells. We think that the confinement of cells in wells is effective to prevent any drifts of measurement points on cell surfaces and thus cells placed in wells are suitable for investigating the long-time relaxation of cell rheology.

\section{Conclusions}

The stress and creep relaxations of mouse fibroblast cells arranged and cultured on a microarray were measured over a timescale of $60 \mathrm{~s}$ by atomic force microscopy with a hydrophobic-coated colloidal probe. The averaged long-time relaxations exhibited a single power-law behavior, with an exponent in the range $0.1-0.4$, which was in good agreement with that of the complex shear modulus of cells observed previously using the force modulation mode in the range of $0.5-200 \mathrm{~Hz}$. This result indicated that the average rheological behavior of cells exhibited a single power-law behavior in time regions from milliseconds to several tens seconds.

\section{Acknowledgments}

We would like to thank Professor B. Fabry for his fruitful comments. This work was supported by Industrial Technology Research Grant Program (2006) from the New Energy and Industrial Technology Development Organization (NEDO) of Japan, Research for Promoting Technological Seeds A (2008) of Japan Science and Technology Agency (JST), and Global COE Program of the Japanese Society for the Promotion of Science (JSPS). 


\section{References}

1) B. Fabry, G. N. Maksym, J. P. Butler, M. Glogauer, D. Navajas, and J. J. Fredberg: Phys. Rev. Lett. 87 (2001) 148102

2) B. Fabry, G. N. Maksym, J. P. Butler, M. Glogauer, D. Navajas, N. A. Taback, E. J. Millet, and J. J. Fredberg: Phys. Rev. E 68 (2003) 041914.

3) L. Deng, X. Trepat, J.P. Butler, E. Millet, K. G. Morgan, D. A. Weitz, and J. J. Fredberg: Nat. Mater. 5 (2006) 636.

4) B. D. Hoffman, G. Massiera, K. M. Van Citters, and J. C. Crocker: Proc. Natl. Acad. Sci. U.S.A. 103 (2006) 10259.

5) K. M. Van Citters, B. D. Hoffman, G. Massiera, and J. C. Crocker: Biophys. J. 91 (2006) 3946.

6) G. Massiera, K. M. Van Citters, P. L. Biancaniello, and J. C. Crocker: Biophys. J. 93 (2007) 3703.

7) M. Balland, N. Desprat, D. Icard, S. Féréol, A. Asnacios, J. Browaeys, S. Hénon, and F. Gallet: Phys. Rev. E 74 (2006) 021911.

8) Atomic Force Microscopy in Cell Biology, ed. B. P. Jena and J. K. H. Horber (Academic Press, New York, 2002) Methods in Cell Biology Vol. 68.

9) V. J. Morris, A. R. Kirby, and A. P. Gunning: Atomic Force Microscopy for Biologists (Imperial College Press, London, 1999).

10) M. Radmacher, R. W. Tillmann, M. Fritz, and H. E. Gaub: Science 257 (1992) 1900.

11) M. Radmacher, R. W. Tillmann, and H. E. Gaub: Biophys. J. 64 (1993) 735. 
12) R. E. Mahaffy. S. Park, E. Gerde, J. Kas, and C. K. Shih: Biophys. J. 86 (2004) 1777.

13) J. Alcaraz, L. Buscemi, M. Grabulosa, X. Trepat, B. Fabry, R. Farre, and D. Navajas: Biophys. J. 84 (2003) 2071.

14) B. A. Smith, B. Tolloczko, J. G. Martin, and P. Grutter: Biophys. J. 88 (2005) 2994.

15) P. Roca-Cusachs, I. Almendros, R. Sunyer, N. Gavara, R. Farre, and D. Navajas: Biophys J 91(2006) 3508.

16) S. Hiratsuka, Y. Mizutani, M. Tsuchiya, K. Kawahara, H. Tokumoto, and T. Okajima: to be published in Ultramicroscopy.

17) H. W. Wu, T. Kuhn, and V. T. Moy: Scanning 20 (1998) 389.

18) E. M. Darling, S. Zauscher, and F. Guilak: Osteoarthritis Cartilage 14 (2006) 571.

19) E. M. Darling, S. Zauscher, J. A. Block, and F. Guilak: Biophys. J. 92 (2007) 1784.

20) T. Okajima, M. Tanaka, S. Tsukiyama, T. Kadowaki, S. Yamamoto, M. Shimomura, and H. Tokumoto: Nanotechnology 18 (2007) 084010.

21) T. Okajima, M. Tanaka, S. Tsukiyama, T. Kadowaki, S. Yamamoto, M. Shimomura, and H. Tokumoto: Jpn. J. Appl. Phys. 46 (2007) 5552.

22) J. J. Fredberg and D. Stamenovic: J. Appl. Physiol. 67 (1989) 2408.

23) J. Hildebrandt: Bull. Math. Biophys. 31 (1969) 651.

24) Y. Mizutani, M. Tsuchiya, S. Hiratsuka, K. Kawahara, H. Tokumoto, and T. Okajima: Jpn. J. Appl. Phys. 47 (2008) 6177. 
25) U. Srinivasan, M. R. Houston, R. T. Howe, and R. Maboudian: J. Microelectromech. Syst. 7 (1998) 252.

26) M. Doms, H. Feindt, W. J. Kuipers, D. Shewtanasoontorn, A. S. Matar, S. Brinkhues, R. H. Welton, and J. Mueller: J. Micromech. Microeng. 18 (2008) 055030.

27) J. L. Hutter and J. Bechhoefer: Rev. Sci. Instrum. 64 (1993) 1868.

28) L. D. Landau and E. M. Lifshiz: Theory of Elasticity (Pergamon Press, Oxford, U.K., 1986).

29) W. N. Findley, J. S. Lai, and K. Onaran: Creep and Relaxation of Nonlinear Viscoelastic Materials with an Introduction to Linear Viscoelasticity (Dover, New York, 1989).

30) K. L. Johnson: Contact Mechanics (Cambridge University Press, Cambridge, U.K., 1987).

31) N. Desprat, A. Richert, J. Simeon, and A. Asnacios: Biophys. J. 88 (2005) 2224.

32) D. R. Overby, B. D. Matthews, E. Alsberg, and D. E. Ingber: Acta Biomater. 1 (2005) 295 .

33) D. Stamenovic', N. Rosenblatt, M. Montoya-Zavala, B. D. Matthews, S. Hu, B. Suki, N. Wang, and D. E. Ingber: Biophys J. 93 (2007) L39. 


\section{Figure captions}

Fig. 1. (Color online) (a) Optical image showing the cantilever on cells arranged and cultured on the wells of microarray. (b) Schematics of stress relaxation and creep measurements using AFM. Several stress relaxation curves for each of five cells were measured at the center of the microarray wells over a time interval of ca. 4 min. Next, creep relaxation measurements were made for the same cells. The stress and creep relaxation curves obtained from single cells were normalized, and the averaged relaxation curves were estimated for each of cells.

Fig. 2. Histograms of adhesion work between cells on the microarray and colloidal probes treated with (a) and without (b) FDTS in force measurements at a constant speed. The adhesion work was estimated from the area of pulling the cantilever between approach and retraction curves. The insets show the adhesion work for different cells with the FDTS-treated probe $(n=800$ cells $)$ and with the untreated probe $(n=1100$ cells).

Fig. 3. Linear plot of the averaged stress (a) and creep (b) relaxation curve for NIH3T3 cells on the microarray, measured by AFM. The insets show the corresponding relaxations on logarithmic axes. Solid lines represent the fit of the power-law function described by eq. (2') for (a) and eq. (3') for (b). 
Fig. 4. Single power-law exponent, $\alpha$, of the averaged stress (closed symbols) and creep (open symbols) relaxations for each cells. The values of $\alpha$ were estimated using eq. (2') for stress relaxation and eq. (3') for creep relaxation. 
Fig.1 Hiratsuka et al. (Color online)

(b)

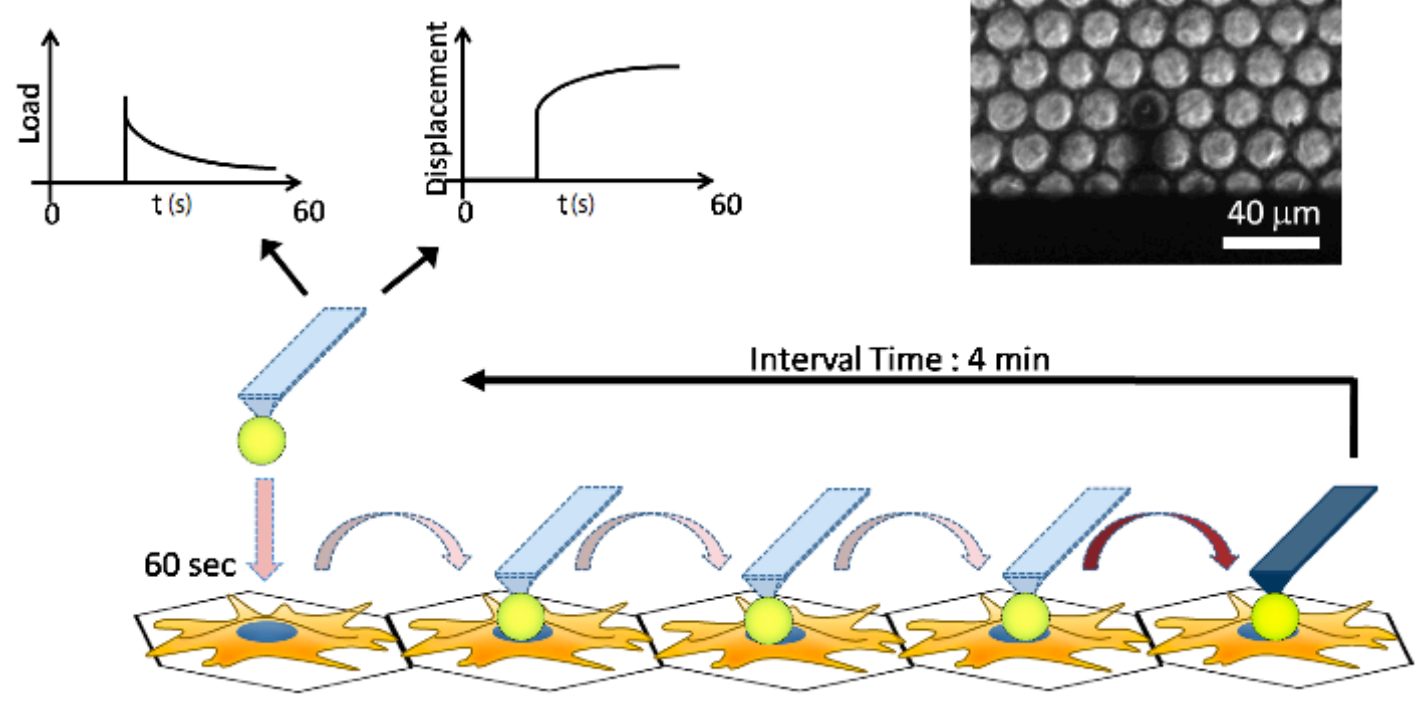


Fig.2 Hiratsuka et al.
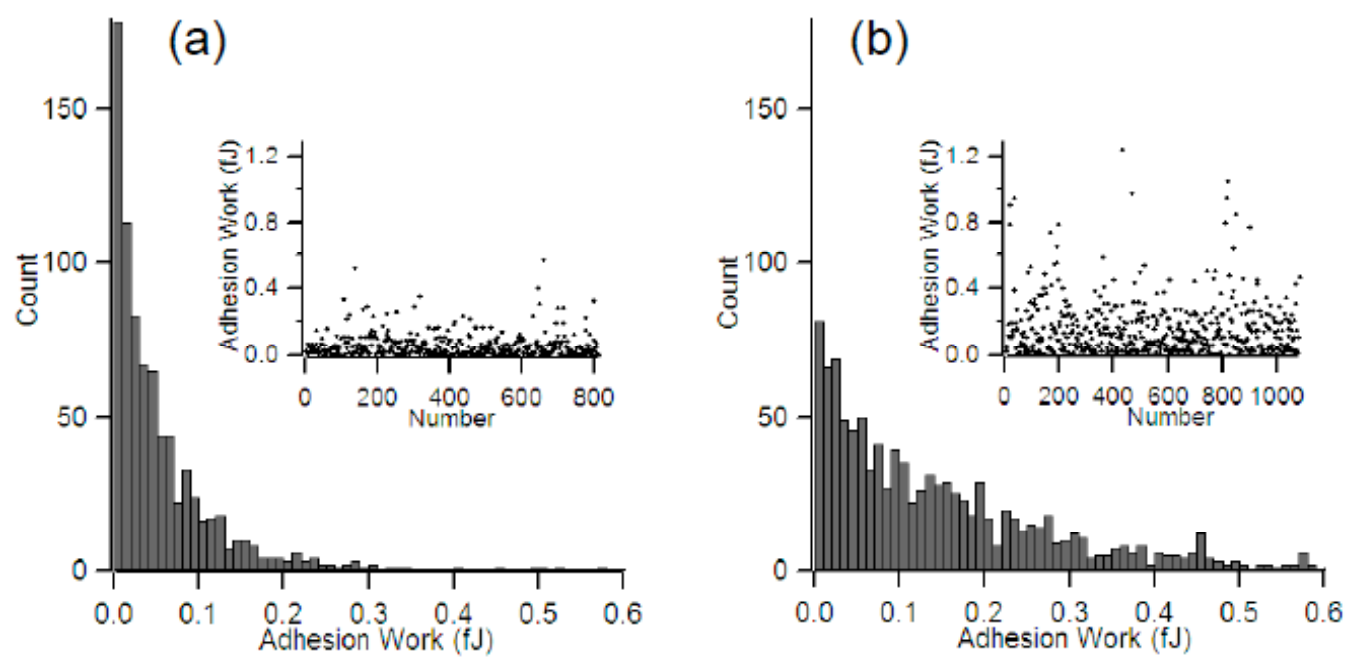
Fig.3 Hiratsuka et al.
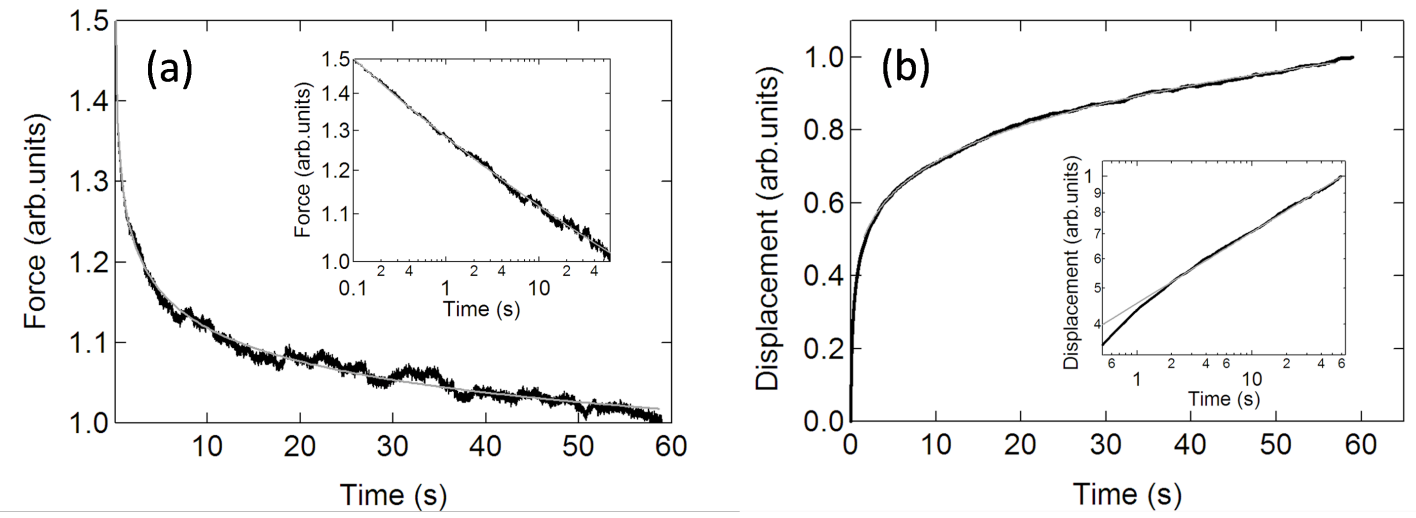
Fig.4 Hiratsuka et al.

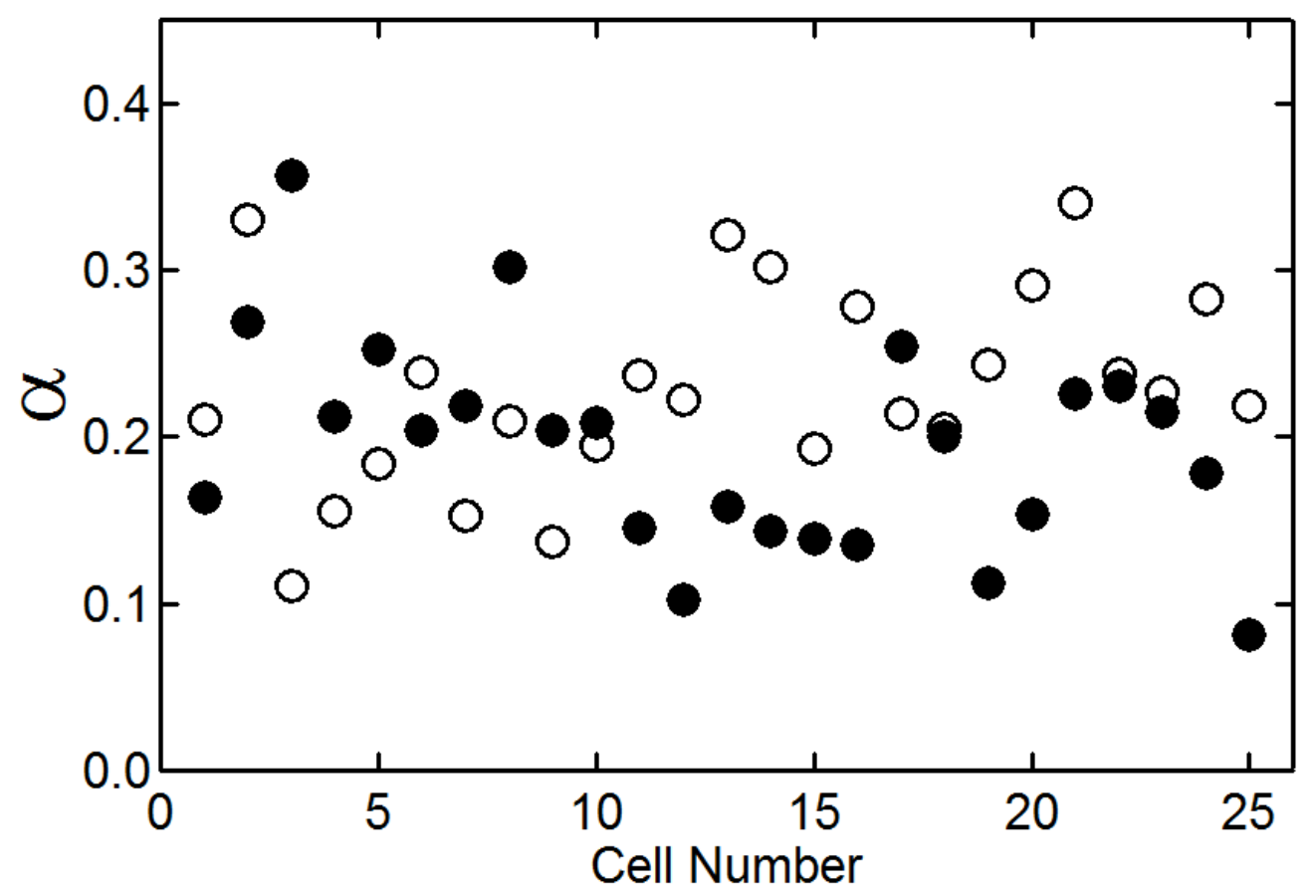

